\title{
Modélisation de composés ordonnés sous irradiation : diagrammes d'équilibre dynamique et évolutions microstructurales
}

\author{
F. SOISSON, P. BELLON et G. MARTIN
}

CEREM-DTM-SRMP, CE de Saclay, 91191 Gif-sur-Yvette cedex, France

\begin{abstract}
To model the behaviour under irradiation of a B.C.C. alloy presenting the B2-A2 order-disorder transitibn, we propose a mean-field approximation of a kinetic model with two atomic exchange mechanisms acting in parallel : thermally activated and forced jumps. By integration of deterministic evolution equations, we show that irradiation can induce the stabilization of a two-phase alloy, while the classical equilibrium phase diagram only displays single phase fields (the B2-A2 transition is second order). Steady-state diagrams are computed : in the two-phase region, antiphase boundaries are found to be unstable with respect to the disordered solid solution and smaller precipitates shrink to the benefit of the larger ones. The different dissolution paths of B2 precipitates are studied.

Résumé: Afin de modéliser le comportement d'un alliage ordonné de structure cubique centrée sous irradiation, nous proposons - dans le cadre d'une approximation de champ moyen un modèle cinétique où deux mécanismes de diffusion atomique opèrent en parallèle: des sauts "balistiques" et des sauts thermiquement activés. Par intégration numérique des équations d'évolution déterministes, nous montrons que l'irradiation peut stabiliser dans un état biphasé un alliage qui n'existe hors irradiation que dans des états monophasés. Des diagrammes d'équilibre dynamique peuvent être construits. Dans le domaine biphasé, nous étudions le mécanisme d'élimination des parois d'antiphases, ainsi que les différents régimes de dissolution des précipités ordonnés.
\end{abstract}

\section{Introduction}

L'irradiation d'un alliage peut accélérer ou même provoquer de nombreuses transitions de phases [1]: amorphisation d'une phase cristalline, précipitation dans une solution solide sous-saturée ou au contraire dissolution de précipités. Dans le cas d'alliages présentant une tendance à l'ordre, l'état du système résulte dans les cas les plus simples - d'une compétition entre le désordre provoqué par les chocs nucléaires et l'accélération de la remise en ordre due à la production de défauts ponctuels en excès [2]. Dans certains cas, cette compétition peut stabiliser des phases qui seraient instables ou métastables hors irradiation [3]. Des lois empiriques ont été proposées pour chaque type de transition, mais l'existence de phénomènes à seuil rend hasardeuse leur extrapolation à des conditions d'irradiation trés différentes de celles pour lesquelles elles ont été établies. Le problème se pose en particulier pour les futurs réacteurs à fusion (neutrons de 14 $\mathrm{MeV}$ ).

Un alliage irradié doit en fait être considéré comme un système dynamique maintenu loin de l'équilibre par une sollicitation extérieure. A l'échelle microscopique, des déplacements forcés ou balistiques d'atomes 
(provoqués par les chocs nucléaires) s'ajoutent aux déplacements thermiquement activés de la diffusion classique. Le système explore l'espace de ses configurations en obéissant à deux dynamiques qui opèrent en parallèle et dont l'une, indépendante de l'état du système et de sa température, tend toujours à l'homogénéiser et à le désordonner, tandis que l'autre tend à le ramener vers son état d'équilibre. L'alliage peut alors se maintenir dans des états stationnaires qui ne seraient pas stables hors irradiațion. Contrairement à une situation d'équilibre thermodynamique (où l'état stable est déterminé uniquement par le minimum d'énergie libre) ces états stationnaires dépendent des mécanismes atomiques de diffusion. Il faut donc utiliser des modèles fondés sur ces processus élémentaires.

Des modèles homogènes de type "cinétique chimique" prenant en compte cette compétition entre deux dynamiques ont longtemps été utilisés pour reproduire les courbes de mise et ordre ou en désordre expérimentales $[4,5]$. Cependant dès qu'une transition devient du premier ordre, une description hétérogène devient indispensable pout étudier les propriétés microstructurales. Dans le cadre d'un modèle simple de diffusion sous irradiation, nous proposons ici une description hétérogène de cinétique déterministe, en l'appliquant au cas des alliages cubiques centrés. Cette méthode permet de construire des diagrammes d'équilibre dynamiques et d'étudier les propriétés des interfaces cohérents dans les alliages biphasés.

On considère un alliage binaire de substitution $A_{c} B_{1-c}$, sur un réseau rigide de structure cubique centrée. Ce réseau peut être décomposé en deux sous réseau ( $\alpha$ et $\beta$ ) dont les sites occupent respectivemént les centres et les sommets des mailles. Hors irradiation et à basse température, l'alliage est ordonné (phase B2): les atomes $A$ se répartissent préférentiellement sur les sites $\alpha$, les atomes $B$ sur les sites $\beta$. Pour chaque composition, le degré d'ordre à longue distance du système peut être caractérisé soit par les concentrations en $A$ sur les deux sous-réseaux (notées $C^{\alpha}$ et $C^{\beta}$ ), soit par la composition moyenne $C$ et le degré d'ordre à longue distance $S=2\left(C^{\alpha}-C\right)=-2\left(C-C^{\beta}\right)$. Une approximation de Bragg-Williams peut alors être utilisée pour modéliser de manière qualitative la transition ordre-désordre B2-A2 [6]. Si on se limite à des interactions entre atomes premiers voisins, la transition est du deuxième-ordre à l'équilibre, avec une température de transition $T_{c}=2 \Omega / k_{b} \times C(1-C)$, où $\Omega=z w^{(1)} / 2$ est l'énergie d'ordre de l'alliage $\left(w^{(n)}=z / 2 \times\left(V_{a a}^{(n)}+V_{b b}^{(n)}-2 V_{a b}^{(n)}\right), z\right.$ est le nombre de coordination $-z=8$ pour la structure cubique centrée - et $V_{i j}^{(n)} l^{a}$ 'énergie de la paire d'atomes $i-j$ situés sur des sites $n^{i e m e s}$ voisins). Dans ce cas seuls des états homogènes (avec un degré d'ordre variant continûment entre 0 et 1 lorsque la température diminue) sont stables. Si l'on tient compte en plus d'interactions aux seconds voisins, la transition peut devenir du premier ordre pour certaines valeur du paramètre $V=w^{(2)} / w^{(1)}$ et de la composition, et on observe alors à l'équilibre la coexistence d'une phase ordonnée et d'une phase désordonnée.

\section{Modèle de diffusion sous irradiation}

Nous modélisons la diffusion sous irradiation de façon très simple, par des échanges directs de paires $A-B$, selon deux mécanismes opérant en parallèle:

- des échanges balistiques induits par les collisions nucléaires, avec une fréquence $\Gamma_{b}$ indépendante de l'état du système et de sa température.

- des échanges thermiquement activés. Dans le cadre des théories de cinétique chimique, l'énergie d'activation est l'énergie nécessaire pour extraire la paire $A-B$ de son environnement (calculée en comptant le nombre de liaisons coupées) et pour l'amener en position de col, où son énergie est $E_{s}$ (en première approximation $E_{s}$ est prise comme une constante indépendante de l'environnement de la paire $A-B$ ).

L'échange d'un atome $A$ occupant le $i^{i e m e}$ site du sous-réseau $\alpha$, avec un atome $B$ occupant le $j^{\text {ieme }}$ site du sous-réseau $\beta$, se produira à une fréquence:

$$
\Gamma_{\alpha \beta}^{i j}=\Gamma_{\alpha \beta}^{t h}+\Gamma_{b}=\nu \exp \left(-\frac{E_{\alpha \beta}^{i \dot{j}}}{k_{b} T}\right)+\Gamma_{b}
$$

et l'échange inverse à la fréquence: 


$$
\Gamma_{\beta \alpha}^{j i}=\Gamma_{\beta \alpha}^{t h}+\Gamma_{b}=\nu \exp \left(-\frac{E_{\beta \alpha}^{j i}}{k_{b} T}\right)+\Gamma_{b}
$$

\section{Description cinétique déterministe}

Afin de suivre l'évolution microstructurale de l'alliage, nous considérons une description hétérogène formée d'un empilement de $N$ mailles cubiques centrées et nous définissons des concentrations locales sur chaque site de ce réseau. Nous nous sommes limités à des problèmes de điffusion à une ou deux dimensions: en accord avec l'approximation de Bragg-Williams ces concentrations correspondent à des moyennes (effectuées dans des plans ou sur des colonnes atomiques perpendiculaires à la direction ou au plan de diffusion) du cristal original à trois dimensions. Avec des interactions entre atomes premiers voisins uniquement, les énergies d'activation des équations (1) et (2) s'écrivent:

$$
E_{\beta \alpha}^{i j}=E_{s}-\sum_{p}^{n n(i)}\left\{C_{p}^{\beta} V_{a a}^{(1)}+\left(1-C_{p}^{\beta}\right) V_{a b}^{(1)}\right\}-\sum_{q}^{n n(j)}\left\{C_{q}^{\alpha} V_{a b}^{(1)}+\left(1-C_{q}^{\alpha} V_{b b}^{(1)}\right)\right\}
$$

(et une expression analogue pour $E_{\alpha \beta}^{j i}$ ), où $C_{q}^{\alpha}$ et $C_{p}^{\beta}$ sont les concentrations atomiques des mailles $q$ et $p$, et où $i$ et $j$ vont de 1 à $N$. Les sommations sont limités aux $n n(i)$ et $n n(j)$ premiers voisins des sites $i$ et $j$. L'évolution de la concentration sur le site $i$ du sous-réseau $\alpha$ est alors donné par:

$$
\frac{d C_{i}^{\alpha}}{d t}=\sum_{j}^{n n(i)}\left\{-\Gamma_{\alpha \beta}^{i j} C_{i}^{\alpha}\left(1-C_{j}^{\beta}\right)+\Gamma_{\beta \alpha}^{j i} C_{j}^{\beta}\left(1-C_{i}^{\alpha}\right)\right\}
$$

Celle de $C_{j}^{\beta}$ est donnée par une expression analogue avec $\alpha$ et $\beta$ inversés.

Pour un système thermique $\left(\Gamma_{b}=0\right)$ les équations $d C_{i}^{\alpha} / d t=0$ et $d C_{j}^{\beta} / d t=0$ font tendre le système vers un état stationnaire qui coïncide avec l'état d'équilibre obtenu en minimisant l'énergie libre calculée dans la même approximation de Bragg-Williams [7]. Pour un système forcé $\left(\Gamma_{b} \neq 0\right)$, l'état de l'alliage est déterminé par la température réduite $T / T_{c}$, la composition moyenne $C$ et une fréquence de sauts forcés réduite:

$$
\gamma=\frac{\Gamma_{b}}{\nu} \exp \left(\frac{E_{s}-E_{o}}{k_{b} T}\right)=\frac{\Gamma_{b}}{\left.<\Gamma_{t h}\right\rangle}
$$

où $E_{o}=z\left\{C V_{a a}^{(1)}+(1-C) V_{b b}^{(1)}+V_{a b}^{(1)}\right\}$ et où $\left\langle\Gamma_{t h}>\right.$ est une fréquence moyenne des sauts thermiques, qui dépend de la température mais qui ne dépend - si la diffusion est modélisée par des échanges directs de paires $A-B-$ ni du flux d'irradiation, ni de la concentration en défauts ponctuels.

L'évolution du système et ses différents états stationnaires sont obtenus par intégration numériques des équations (4), en utilisant des conditions aux limites périodiques, et en partant de différents états initiaux $\left\{C_{i}^{\alpha}(t=0), C_{j}^{\beta}(t=0)\right\}$. Typiquement, nous avons utilisé des réseaux comprenant de $N=40$ à $N=400$ mailles pour les problêmes à une dimension, et $N=40 \times 40$ à $N=80 \times 80$ pour les problèmes à deux dimensions. Les intégrations numériques ont été réalisées par une méthode de Runge-Kutta d'ordre 4 (avec adaptation controlée du pas de temps), l'erreur relative sur les concentrations étant maintenue inférieure à $10^{-6}$ à chaque pas et l'état stationnaire étant supposé atteint pour des dérivée par rapport au temps inférieures à $10^{-8}$.

Il est à noter que ce type de modèle souffre des inconvénients propres à toute description déterministe: lorsque pour les mêmes conditions d'irradiation plusieurs états sont localement stables, leur stabilité relative ne peut pas être comparée. Toutefois, d'autres techniques, prenant en compte les fluctuations $[8,9,10]$ permettent de confirmer les prévisions de cette approche. 


\section{Diagrammes d'équilibres dynamiques}

A l'équilibre, avec des interactions limitées aux premiers voisins $\left(w^{(2)}=0\right)$, la transition A2-B2 est du deuxième ordre dans tout le domaine de composition. Sous irradiation, la transition peut devenir du premier ordre pour une valeur critique de $\gamma$. Des expériences d'irradiation d'alliages $F e-A l$ irradiés aux électrons de $1 \mathrm{MeV}$ confirment cette prédiction [11]. La ligne tricritique est donnée par [9]:

$$
\begin{aligned}
& \frac{T^{*}}{T_{c}}=\frac{8 C(1-C)}{3+\sqrt{9-24 C(1-C)}} \\
& \gamma^{*}=\frac{\sqrt{9-24 C(1-C)}+1}{2}
\end{aligned}
$$

Au-delà de cette ligne, un alliage biphasé peut être stabilisé où une phase ordonnée coexiste avec une phase désordonnée appauvrie en espèce minoritaire. Cet état biphasé est bien stabilisé par l'irradiation puisque le diagramme de phases d'équilibre ne comprend que des domaines monophasés. Un exemple de diagramme des états stationnaires (pour une valeur $\gamma=4.55$ ) est donné sur la figure 1 , sur laquelle sont reportées les limites du domaine biphasé et les limites au-delà desquelles les états homogènes (ordonnés ou désordonnés) deviennent instables. Dans le domaine biphasé, la proportion des deux phases obéit à la règle du bras de levier: une modification de la composition moyenne $C$ entraîne une modification de cette proportion mais n'affecte pas la composition de chacune des deux phases.

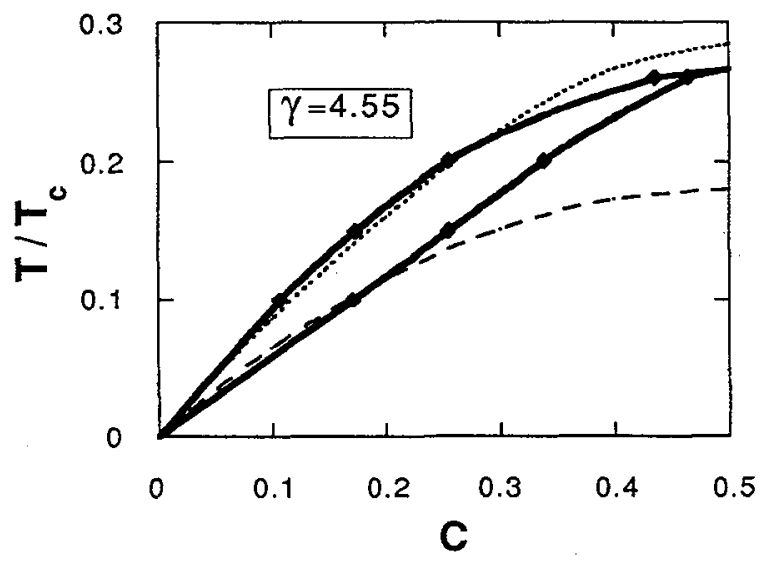

Figure 1: Diagramme des états stationnaires pour $\gamma$ constant $(\gamma=4.55)$

Limites du domaine biphasé
$\ldots \ldots-$ Limite d'instabilité de la solution homogène désordonnée
$\ldots . \ldots \ldots$

Comme le montre l'équation (5) le paramètre $\gamma=\Gamma_{b} /\left\langle\Gamma_{t h}\right\rangle$ est fonction de $\Gamma_{b}$ et de la température. De plus, si on considère un modèle de diffusion sous irradiation plus réaliste (diffusion par l'intermédiaire des défauts ponctuels au lieu du mécanisme d'échange direct décrit plus haut) $\left\langle\Gamma_{t h}\right\rangle$ est proportionnelle à la concentration en défauts ponctuels qui dépend elle-même de la température et du flux d'irradiation. Dans ce cas, en supposant qu'à l'état quasi-stationnaire la concentration en lacunes et en interstitiels est limitée par la recombinaison des paires de Frenkel, $\gamma$ est donné par [7]: 


$$
\gamma=g\left(\frac{\Gamma_{b}}{\Gamma_{v}}\right)^{1 / 2}
$$

où $g$ est un facteur géométrique, et $\Gamma_{v}$ la fréquence moyenne de sauts des lacunes

$$
\Gamma_{v}=\Gamma_{v}^{o} \exp \left(-\frac{E_{v}^{m}}{k_{b} T}\right)
$$

$E_{v}^{m}$ étant l'énergie de migration des lacunes. Avec $\gamma_{0}=g\left(\Gamma_{b} / \Gamma_{v}^{o}\right)^{1 / 2}$,on obtient

$$
\gamma=\gamma_{o} \exp \left(\frac{E_{v}^{m}}{2 k_{b} T}\right)
$$

Quand la population de défauts atteint un état quasi-stationnaire [12], on montre facilement qu'une expression analogue reste valable, en remplacant dans l'équation (10) l'énergie de migration des lacunes par l'énergie de migration des interstitiels $E_{i}^{m}$. Les conditions d'irradiation sont alors définies par $T / T_{c}$, $C$ et $\gamma_{0}$, et un diagramme des états stationnaires à $\gamma_{0}$ constant peut être calculé, comme dans les conditions expérimentales où le flux d'irradiation (qui détermine $\Gamma_{b}$ ) et la température sont fixés de manière indépendante. Un tel diagramme est reproduit sur la figure 2 avec $\gamma_{0}=10^{-5}$ et une valeur typique $E_{d}^{m}=0.5$ $\mathrm{eV}$ pour l'énergie de migration de défauts.

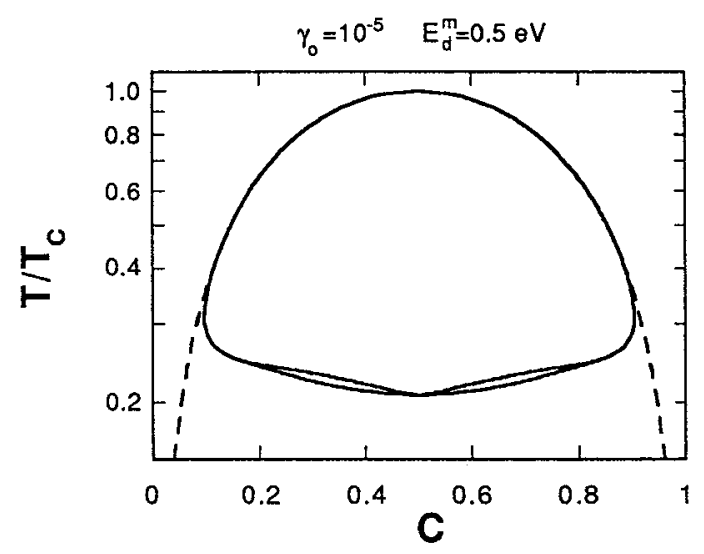

Figure 2: Diagramme des états stationnaires (lignes continues) pour $\gamma_{0}$ constant $\left(\gamma_{0}=10^{-5}\right)$, énergie de migration de défaut $\mathrm{E}_{\mathrm{d}}^{\mathrm{m}}=0.5 \mathrm{eV}$, et diagramme de phases à l'équilibre (lignes discontinues).

\section{Evolutions de microstructures sous irradiation}

Les équations cinétiques (4) nous permettent de suivre l'évolution du système à partir d'un état qui n'est pas stationnaire. A titre d'exemple nous évoquerons ici la réaction des précipités ordonnés à l'introduction d'une paroi d'antiphase (comme se serait le cas à la suite de son cisaillement par une dislocation), ainsi que les différents régimes de dissolution de ces précipités sous irradiation. 


\subsection{Redistribution des précipités antiphasés}

Considérons tout d'abord un alliage biphasé stabilisé par l'irradiation (son point représentatif $C=0.3, T / T_{c}=$ $0.2, \gamma=4.55$ se trouve dans le domaine biphasé de la figure 1). Sur la figure 3(a) on peut suivre son évolution après l'introduction d'une paroi d'antiphase (PAP) dans la phase ordonnée, le long d'une direction $\langle 110\rangle$. On observe la croissance d'une phase désordonnée le long de la PAP. Les deux précipités se "repoussent" mutuellement par un mécanisme de dissolution et de redéposition. Le plus petit des deux précipités fini par disparaître au bénéfice du plus grand. Au cours du processus, on note un déplacement du centre du précipité ordonné. L'évolution est qualitativement la même avec une PAP conservative $\{110\}$ ou non conservative $\{100\}$.

Sous irradiation, les défauts ponctuels s'accumulent sous forme de boucles de dislocations qui, au cours de leur croissance, peuvent cisailler les précipités ordonnés. Potter et al. [13] ont ainsi observés le cisaillement de précipités ordonnés $\gamma^{\prime}$ dans un alliage $\mathrm{Ni}-\mathrm{Al}\left(12 \%\right.$ at. $\mathrm{Ni}$ ) irradié aux ions $\mathrm{Ni}^{+}$. Ce cisaillement est suivi d'une dissolution des précipités que Potter interprète en termes de couplage entre flux d'atomes de soluté et flux de lacunes vers les boucles de dislocations [14]. Notre modèle, où les défauts ponctuels n'interviennent pas, fourni une autre explication possible.

Afin de comparer ce comportement avec celui d'un système thermique, il nous faut introduire des interactions aux seconds voisins: pour certaines valeurs du paramètre $V=w^{(1)} / w^{(2)}$, un alliage biphasé peut être stable hors irradiation [15]. Les équations (3) et (4) peuvent alors être généralisées pour prendre en compte les potentiels de paires au seconds voisins et les mêmes techniques numériques être utilisées pour le calcul des états stationnaires. A des températures suffisamment basses (où les précipités sont facettés) après son introduction dans un tel système (avec $V=-4 / 3, T / T_{c}=0.25, \gamma=0$ ), une PAP tend à s'orienter dans une direction $\langle 100\rangle$ (figure 3(b)). Si la PAP est introduite directement le long de cette direction, on n'observe pratiquement aucune évolution: le système reste piégé dans un état métastable. A une température plus élevée, $\left(T / T_{c}=0.5\right)$ le précipité ordonné s'arrondit et l'évolution du système après l'introduction d'une PAP devient similaire à celle de la figure 3(a) : l'isotropie de la tension superficielle semble donc jouer un rôle important dans le mécanisme d'élimination des PAP.

Sous irradiation, la limite à haute température du domaine biphasé (fig. 1) est abaissée lorsque le paramètre $\gamma$ augmente: ainsi, augmenter $\gamma$ en gardant la température et la composition constante provoque un effet analogue à une élévation de température. De fait l'évolution de deux systèmes $\left(T / T_{c}=0.5\right.$ et $\left.\gamma=0\right)$ et $\left(T / T_{c}=0.2\right.$ et $\left.\gamma=4.55\right)$ est similaire. L'effet des sauts ballistiques peut être vu comme une augmentation de la température effective de l'alliage, ce qui rappelle le règle de la température effective introduite par Martin [16] pour rationaliser les phénomènes de démixtion sous irradiation.

\subsection{Régimes de dissolution des précipités ordonnés}

De nombreuses expériences ont montré que dans certains alliages des précipités ordonnés pouvaient être dissous par irradiation. La question s'est posée de savoir si dans de tels cas le précipité se désordonne avant de se dissoudre ou s'il reste ordonné jusqu'à sa disparition. Dans notre modèle, ces deux types de comportement peuvent être observés selon les conditions d'irradiation.

Les figures 4(a) et 4(b) représentent l'évolution de l'alliage, initialement dans un état stationnaire biphasé stabilisé par l'irradiation, après une brusque variation d'un des paramètres de contrôle (ici la température). Si le point représentatif du système $\left(C, T / T_{c}, \gamma\right)$ sort du domaine biphasé mais reste sous la limite d'instabilité de la solution homogène ordonnée, le précipité reste ordonné à cœur jusqu'à sa disparition totale, qui s'opère par progression de l'interface matrice/précipité (fig.4(a)). En revanche, au-dessus de cette limite, le précipité est d'abord désordonné "en bloc", avant que la concentration ne s'homogénéi se dans l'alliage (Fig.4(b)). Remarquons que la position et la définition même des limites d'instabilité sont étroitement liées à l'approximation de champ moyen utilisée (à la façon de la ligne spinodale dans la théorie de la décomposition spinodale). Des simulations Monte Carlo montrent cependant que ces deux types de comportement sont effectivement observables [17]. 
(a)
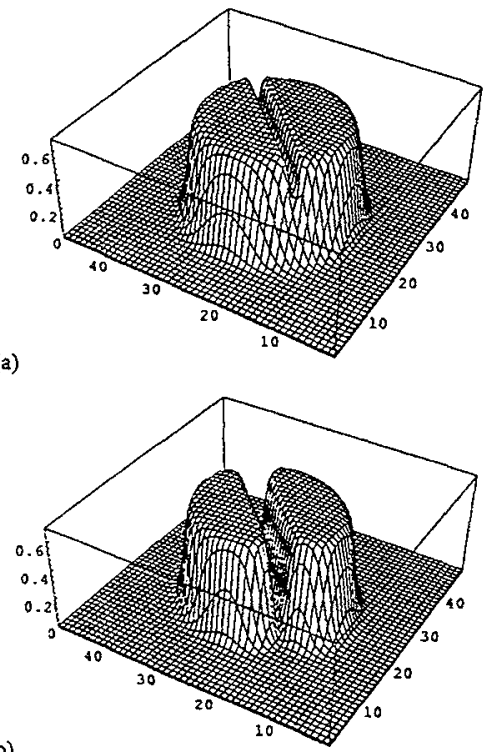

(b)

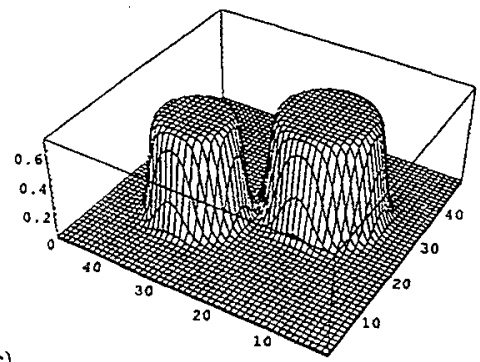

(c)

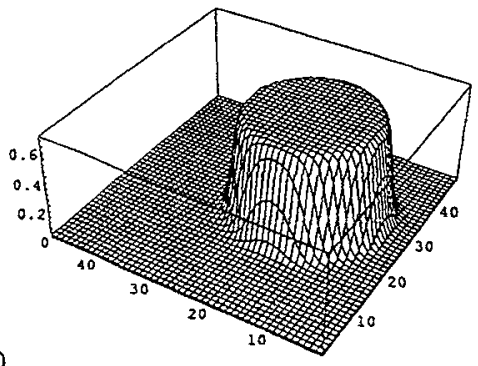

(d)

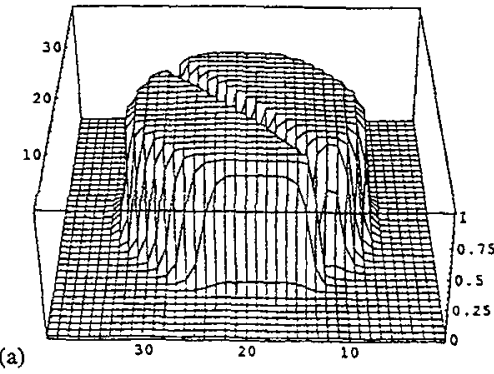

(b)

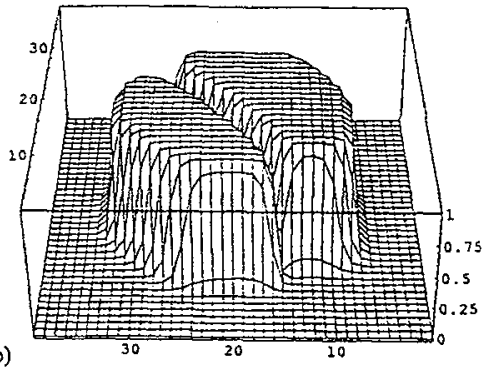

(c)

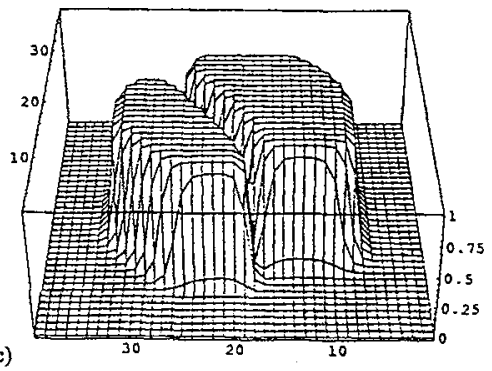

(d)

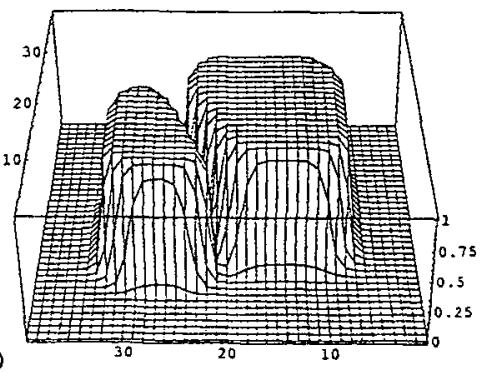

Figure 3: Evolution d'un précipité ordonné après l'introduction d'une paroi d'antiphase conservative. La valeur absolue du degré d'ordre est représentée pour chaque maille du réseau.

(a) Colonne de gauche: système forcé $\mathrm{T} / \mathrm{T}_{\mathrm{c}}=0.2$ et $\gamma=4.55$

En unité de temps arbitraires: a) $\mathrm{t}=0.01$, b) $\mathrm{t}=1, \mathrm{c}$ ) $\mathrm{t}=300$ et $\mathrm{d}$ ) $\mathrm{t}=1000$

(b) Colonne de droite: système thermique $\mathrm{T} / \mathrm{T}_{\mathrm{c}}=0.25$ et $\gamma=0$

En unité de temps arbitraires: a) $\mathrm{t}=10$, b) $\mathrm{t}=1760$, c) $\mathrm{t}=2660$ et d) $\mathrm{t}=8960$ 

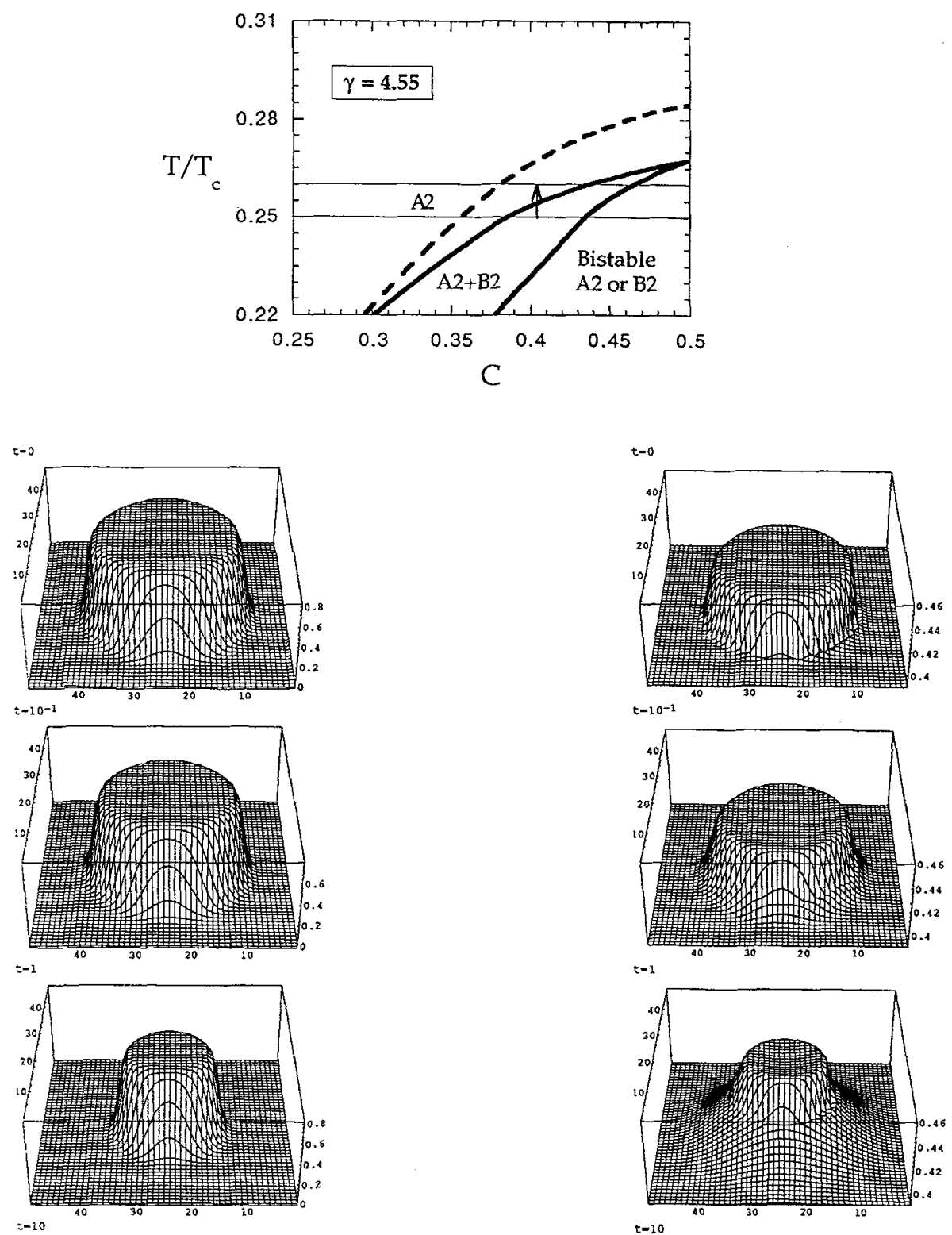

$t=1$
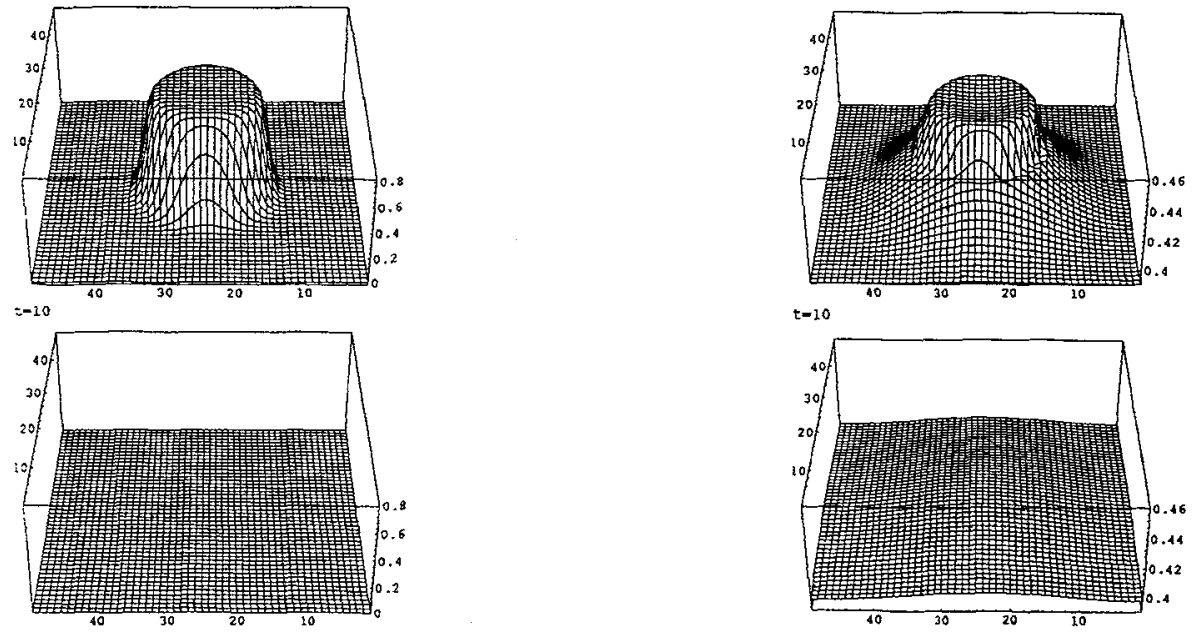

Figure 4(a): Dissolution un precipité ordonné B2 avec une température d'irradiation située au-dessous de la spinodale de désordre $\left(\mathrm{T} / \mathrm{T}_{\mathrm{C}}=0.26\right)$. Evolution du degré d'ordre $\mathrm{S}$ (colonne de gauche) et de la concentration $\mathrm{C}$ (colonne de droite) dans un réseau de $50 \times 50$ cellules cubiques centrées. 

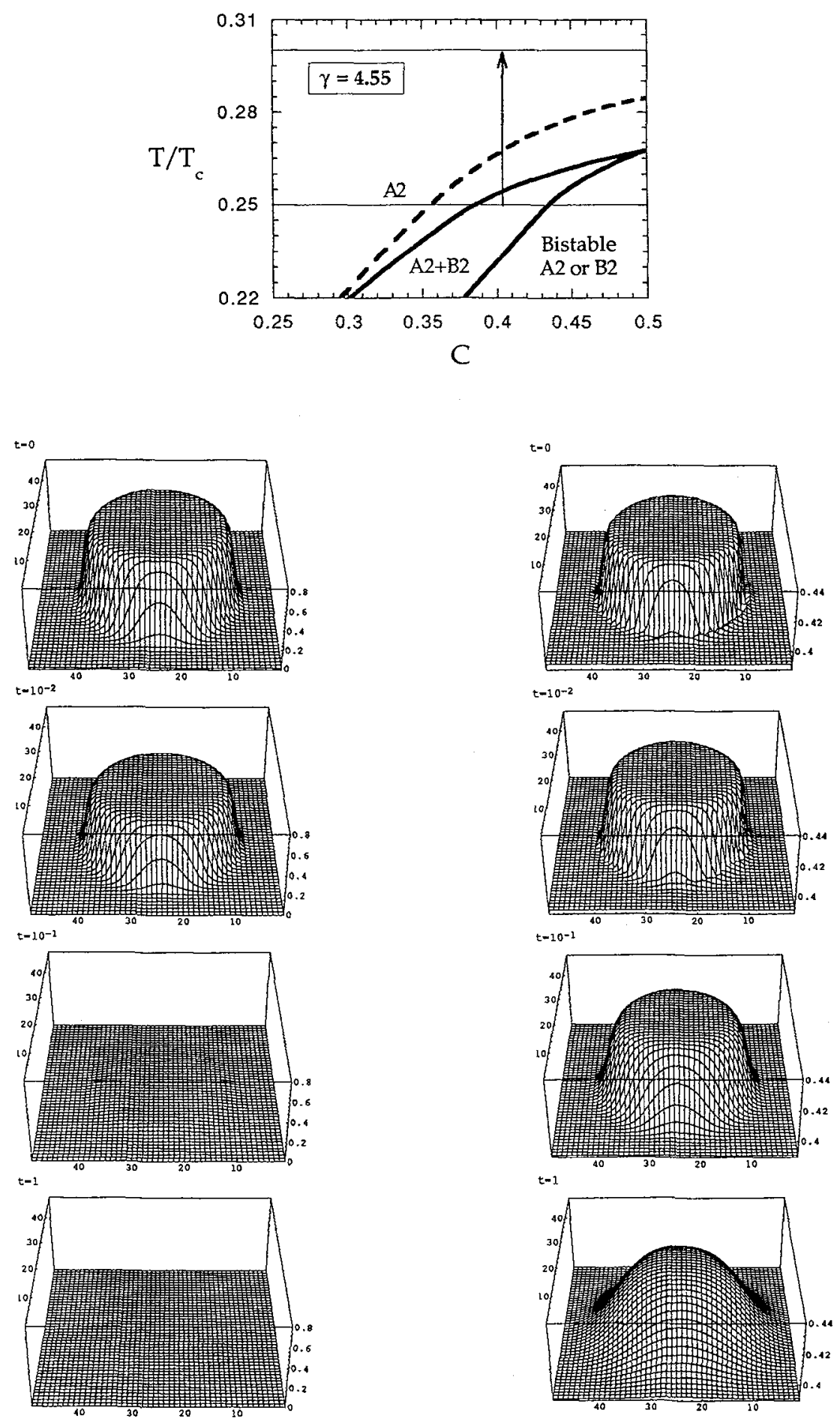

Figure 4(b): Dissolution un precipité ordonné B2 avec une température d'irradiation située au-dessus de la spinodale de désordre $\left(\mathrm{T} / \mathrm{T}_{\mathrm{C}}=0.3\right)$. Evolution du degré d'ordre $\mathrm{S}$ (colonne de gauche) et de la concentration $\mathrm{C}$ (colonne de droite) dans un réseau de $50 \times 50$ cellules cubiques centrées. 


\section{Conclusion}

Nous avons modélisé le comportement d'alliages ordonnés cubiques centrés sous irradiation par intégration numérique d'équations de cinétique déterministe établies dans le cadre d'une approximation de champ moyen. L'irradiation peut stabiliser des états biphasés dans des systèmes n'existant à l'équilibre thermodynamique que dans des états homogènes. Nous avons étudié quelques propriétés des interfaces de ces systèmes: les parois d'antiphases s'éliminent par un mécanisme de dissolution et de redéposition et selon les conditions d'irradiation les précipités se désordonnent à coeur puis se dissolvent, ou bien se dissolvent dans l'état ordonné par migration de l'interface.

\section{References}

[1] K. C. Russell, in Phase Stability Under irradiation, Progress in Materials Science (Pergamon, Oxford, 1984).

[2] E. M. Schulson, J. Nucl. Mat 83 (1979) 239.

[3] S. Banerjee, K. Urban et M. Wilkens, Acta Metall. 32 (1984) 299.

[4] S. Banerjee et K. Urban, Phys. Stat. Sol. (a) 81 (1984) 145.

[5] K.-Y. Liu et P. Wilkes, J. Nucl. Mat 87 (1979) 317.

[6] H. Ackermann, G. Inden et R. Kikuchi, Acta Metall. 37 (1989) 1.

[7] P. Bellon et G. Martin, Phys. Rev. B 39 (1989) 2403.

[8] P. Bellon, Phys. Rev. B 45 (1992) 7517.

[9] F. Soisson, P. Bellon et G. Martin, Phys. Rev. B 46 (1992) 11332.

[10] E. Salomons, P. Bellon, F. Soisson et G. Martin, Phys. Rev. B 45 (1992) 4582.

[11] F. Soisson, P. Dubuisson et P. Bellon, en préparation.

[12] R. Sizman, J. Nucl. Mat 69 \& 70 (1978) 386.

[13] D. I. Potter et A. W. McCormick, Acta Metall. 27 (1979) 933.

[14] D. I. Potter et H. Wiedersich, J. Nucl. Mat 83 (1979) 208.

[15] G. Inden, Acta Metall. 22 (1974) 945.

[16] G. Martin, Phys. Rev. B 30 (1984) 1424.

[17] F. Soisson, P. Bellon et G. Martin, en préparation. 